\title{
Biobehavioral Pathways Underlying Spousal Health Dynamics: Its Nature, Correlates, and Consequences
}

\author{
Christiane A. Hoppmann ${ }^{a}$ Denis Gerstorf ${ }^{b}$

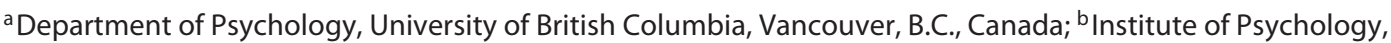 \\ Humboldt University, Berlin, Germany
}

\author{
Key Words \\ Couples · Physical activity · Stress · Collaborative problem \\ solving $\cdot$ Daily-life dynamics
}

\begin{abstract}
Marriage is a very special relationship that has a high potential to influence physical and mental health throughout the adult lifespan and into old age. In this viewpoint, we propose a model that outlines plausible biobehavioral pathways that may underlie previously established spousal interrelations in long-term health trajectories and discuss specific resources that may facilitate favorable outcomes for everyone involved. Specifically, we focus on spousal associations in physical activity as an important health behavior and in stress-related processes as a key daily-life mechanism, which both reveal effects that may accumulate over time to impact longer-term health outcomes. We also consider spousal resources such as collaborative problem solving and joint goals as psychological variables that characterize the dynamics within a given marriage. We conclude by discussing areas in theory and research that are ripe for further consideration and lay out target questions for future inquiry.
\end{abstract}

(c) 2014 S. Karger AG, Basel

\section{KARGER}

(c) 2014 S. Karger AG, Basel

0304-324X/14/0605-0458\$39.50/0

E-Mail karger@karger.com

www.karger.com/ger
'It takes two to tango' is an expression that is commonly used to describe the social dynamics in romantic relationships. Importantly, for many adults marriage is the most central relationship and it has long been known that risks for morbidity and mortality are considerably lower among married as compared to unmarried individuals $[1,2]$. Yet, marriage per se does not guarantee good health. In fact, adults in unhappy marriages face the same health burden as people who are single $[1,3]$. Interestingly, most research investigating the impact of marriage on health has focused on the individual as the unit of analysis (e.g., married individuals on average outlive those who are not married [1]), thereby limiting our ability to better understand the spousal dynamics and mutual influences that occur within a given marriage and link them to aging outcomes $[2,4]$. However, recent research using long-term longitudinal data from both spouses has started to demonstrate that physical and mental health wax and wane in association with the respective spouse over periods of years and decades $[5,6]$.

In this viewpoint, we present a model of spousal interrelations in health that unpacks some of the underlying social dynamics and biobehavioral pathways (fig. 1). First, we outline two biobehavioral pathways that may contribute to previously observed links between spouses

Christiane A. Hoppmann

Department of Psychology, University of British Columbia 2136 West Mall

Vancouver, BC V6T 1 Z4 (Canada)

E-Mail choppmann@ @sych.ubc.ca 


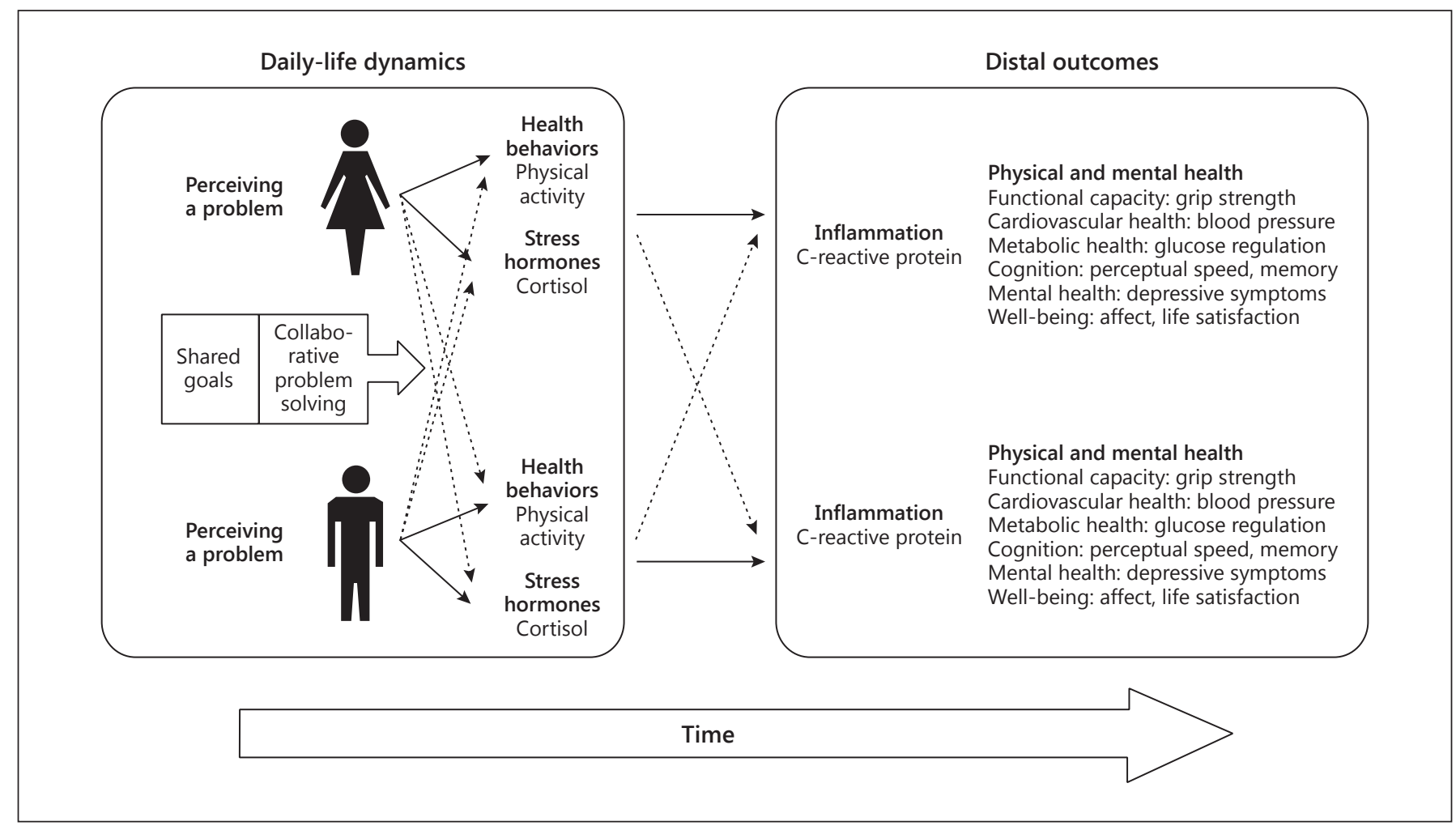

Fig. 1. Working model of spousal interrelations in health.

in long-term health outcomes. Specifically, we focus on spousal associations in physical activity as an important everyday health behavior and on cortisol as a stress hormone that indexes daily-life problems. We will then move on to characterize spousal resources that facilitate how spouses can master the challenges they face as part of their day-to-day lives and engage in healthy lifestyles, thereby contributing to positive long-term health trajectories for everyone involved. Specifically, we will highlight research on collaborative problem solving and joint goals between spouses to illustrate this point. We close by highlighting a number of central objectives that should guide theory and research with implications for behavioral prevention and intervention programs.

\section{Spousal Health Behaviors: The Sample Case of Physical Activity}

Health behaviors represent a very broad category that includes health-compromising activities such as smoking or alcohol abuse as well as health-enhancing activities such as physical activity or fruit and vegetable consumption [7]. For the purpose of the present viewpoint and in keeping with our metaphor, we focus on physical activity, which has been identified as one of the highest priorities for health promotion internationally due to its broad health benefits [8]. For example, physical activity has been shown to reduce the risk of many chronic diseases that increase with aging, such as cardiovascular disease, stroke, type 2 diabetes, and cognitive decline, and it is also associated with higher well-being $[8,9]$. In the following, we argue that health behaviors in general and physical activity in particular represent a core pathway that contributes to spousal interrelations in physical and mental health in middle-aged and older couples.

Physical activity is defined as bodily movement that happens through muscle contraction leading to abovebasal energy expenditure [10]. This definition of physical activity is intentionally broad and reflects that physical activity does not necessarily have to happen in the form of structured exercise. In fact, physical activity also includes activities whose primary purpose is something other than physical activity such as utilitarian walking, 
gardening, heavy household work, or socializing. Physical activity guidelines recommend that adults accumulate at least 150 min of moderate to vigorous intensity physical activity per week in at least 10 -min bouts to achieve substantial health benefits [10]. Engaging in physical activity is very inexpensive considering the costs that an early manifestation of disease would create, but unfortunately the majority of older adults do not meet physical activity guidelines [8]. This has created a pressing need to identify key psychosocial factors that keep middle-aged and older adults from doing what they know is good for them.

Most models of health behavior change focus on the individual [7]. This makes sense given the prominent role of individual goals and intentions as key predictors of health behaviors. However, there is reason to expect that physical activity is tightly linked in couples. For example, there is empirical evidence that the daily-life activities of spouses are closely interrelated [11]. This can be both a blessing and a curse. On the upside, spouses may motivate each other to be physically active so that when one spouse leaves the house and goes on a walk, the other is often involved as well. On the downside, barriers to physical activity are very common in old age. For example, fear of falling may cause an older adult to be afraid to leave the house, thereby reducing physical activity. Such a scenario may not only lead to reduced physical activity in the spouse experiencing the fear, but actually impact the entire couple (e.g., the other spouse stays at home, too, resulting in reduced physical activity in both partners). It thus seems timely to examine physical activity engagement in the context of marriage to better understand the social resources and obstacles to this key health behavior.

\section{Target Questions for Future Research}

There are a number of issues that remain to be addressed to move the field forward. In line with recent research targeting physical activity promotion in clinical samples such as cancer patients, aging research needs to address the spousal mechanisms that govern health behaviors by extending individual-centered models of health behavior change to the couple level [12]. Recent evidence on the role of dyadic planning on exercise promotion in prostatectomy patients and their partners provides empirical evidence that patients benefit if they plan together [12]. Similarly, recent extensions of self-efficacy models also provide a fruitful theoretical backdrop to new spousal approaches by taking into account not just indi- viduals' own beliefs in their capability to become more physically active, but also incorporate self-efficacy beliefs of one's spouse as well as joint relationship-specific selfefficacy, such as the belief to increase physical activity with the help of one's spouse [13]. Such endeavors are not only going to inform research on physical activity, but will also have implications for other key health promoting (e.g., fruit and vegetable consumption) and health compromising (e.g., smoking) behaviors. While dyadic planning or social self-efficacy promise to provide interesting roads for further inquiry, it is also important to recognize that this does not preclude the possibility that spouses may also hinder each other from adopting healthier lifestyles. For example, an older adult who intends to quit smoking and who is married to a heavy smoker is likely going to encounter more obstacles when trying to change her behavior than an older adult whose partner does not smoke.

Furthermore, recent methodological advances such as accelerometers and GPS which is used for tracking people's geographic location throughout the day may help push the envelope to go beyond an examination of data that is based on self-reported physical activity to capture intensity of physical activity as well as life-space mobility [14]. Such technology might also help disentangle some of the mechanisms linking physical activity with physical and mental health benefits. For example, it would be intriguing to examine if it is the actual physiological processes resulting from vigorous physical activity (as captured by accelerometers) that contribute to good health or if the health benefits of physical activity are in fact related to older adults leaving their own home to engage in the community (as captured by GPS monitors). Depending on the outcome, very different recommendations for interventions may emerge. For example, if it was all about intensity of movement, then interventions may focus on strength and balance exercise. If it were about life-space mobility, then an important goal might be to help older adults with getting around in their communities.

Finally, too little is known about specific daily-life barriers to physical activity in older adults. To date, many models of health behavior change that have been developed for younger samples capitalize on goals and motivational processes as important factors governing physical activity [7]. However, it is increasingly recognized that emotional and cognitive factors are also related to physical activity in older adults. Specifically, there is reason to believe that emotional factors such as fear of falling are central barriers to physical activity engagement in seniors [15]. Furthermore, cognitive challenges such as prospec- 
tive memory failures that hamper the implementation of physical activity goals might explain at least in part why some older adults do not translate their goals into action and remain relatively inactive [16]. Understanding the different types of challenges associated with physical activity in older adults (e.g., emotional and/or cognitive barriers) would provide much needed information because such insights are important prerequisites for developing age-appropriate behavioral interventions. One illustrative example of overcoming an emotional barrier to physical activity engagement would be to help older adults to come to grips with and manage feelings of anxiety associated with the fear of falling. An illustrative example of a cognitive training would be to develop programs that guide older adults' attention to information that is relevant for physical activity engagement so that they more readily recognize good opportunities to act.

\section{Spousal Problems and Stress in Older Couples: The Sample Case of Stress Hormones}

Older adults may not only encounter problems with their own or their partner's physical activity, but health issues in general as well as social problems are relatively common stressors in old age [17]. In the following, we outline some of the psychological and biological processes that may follow the appraisal of a problem or stressor in old age. Therein, special attention will be paid to reasons as to why such effects may go beyond the individual experiencing the problem to impact the respective spouse (fig. 1).

Research from unrelated individuals shows that daily problems elicit negative emotions, but the effect often goes beyond alterations in emotional states to also affect biological stress responses [18]. Cortisol for example is a stress hormone that is secreted when the hypothalamicpituitary-adrenal (HPA) axis is activated, and it can be reliably measured in saliva [18]. The ease of salivary collection and the predictable diurnal pattern of cortisol have made this hormone very popular in psychological research. Although there are relatively few studies examining daily-life variability in cortisol in old age, there is evidence of meaningful associations between psychological stressors and daily cortisol in older adults [18]. As noted below, there are several plausible pathways that may link daily stress and longer-term physical and mental health outcomes in old age.

Acute activations of the HPA axis whose final product is cortisol are generally seen as adaptive because they mo- bilize the necessary energy to deal with a given problem [19]. However, chronic activations of the HPA axis can lead to wear and tear and set the stage for alterations in other bodily systems [19]. For example, prolonged exposure to high doses of cortisol may trigger counterregulatory responses such as downregulation of glucocorticoid receptors in various tissues, which desensitizes them to the effects of cortisol [20]. This too is an adaptive response. At the same time, it may also change the dynamics between the different cells of the immune system in such a way that it ultimately facilitates low-grade persistent inflammation [20]. Importantly, chronic activation of the HPA axis in combination with inflammatory processes may have downstream consequences and place an older adult at increased risk for declines in functional capacity, metabolic health, cognition, and mental health [19].

Why do we need to consider spouses when trying to better understand associations between daily stress and longer-term health outcomes in old age? On the positive side, there is evidence that older adults focus on goals that involve close others and that this increased focus on socioemotional goals constitutes an important source of well-being - if things go well [21]. However, by the same token, socioemotional goals may also make an older adult more vulnerable to the experience of stress, for example if close others such as spouses experience a problem. There are at least two plausible mechanisms that may contribute to how problems and stress of one partner affects the other partner. First, if older spouses set joint goals or goals that involve each other [22], then problems experienced by one spouse automatically impact the other spouse as well (e.g., because neither partner will accomplish their goal). Second, stress may be transmitted between spouses [23]. Hence, by virtue of their increased focus on socioemotional goals, older spouses may have more permeable boundaries regarding the respective partner's stress and be more susceptible to the transmission of stress. Older spouses whose partner experiences a problem may therefore be more likely to also experience increased stress, especially if that problem relates to joint goals.

\section{Target Questions for Future Research}

There are a number of additional plausible pathways beyond those indexed by cortisol that ought to be examined when investigating spousal interrelations in stressrelated processes. Specifically, it would be important to ask if spousal associations can also be observed in other 
physiological parameters such as immune functioning, responses to vaccination, or susceptibility to infectious diseases. Furthermore, research examining the role of marital quality on health outcomes in younger samples points to the need to better understand how specific social stressors such as partner conflict may impact cardiovascular reactivity and potentially other stress hormones beyond cortisol such as catecholamines [24].

In addition, it seems timely to investigate the interactive effects between specific stress hormones such as cortisol and other neuropeptides like oxytocin or vasopressin [25]. For example, research with younger couples points to the important role of intimacy and oxytocin in buffering the association between stress exposure and cortisol [26]. It would therefore be interesting to extend this line of research to older couples taking into account age-related changes in biological systems [27]. It would also be interesting to extend experimental paradigms that have been developed for younger couples to examine, for example, if the release of oxytocin triggered by a partner massage can undo the link between stress exposure and cortisol.

Finally, there is experimental evidence pointing to the moderating role of physical activity on stress exposurestress reactivity associations. For example, recent research from a young adult female sample shows that women who exercised more displayed less pronounced heart rate reactivity and cortisol responses when confronted with a well-defined social laboratory stressor than women who were physically inactive [28]. Findings like these further point to the need to bring together lines of research that involve health behaviors and those that involve stress-related processes. Taken together, a couples' approach helps us to better understand how daily problems and stress may be intertwined with close others such as spouses. Importantly, spousal health dynamics should not only be seen as a vulnerability factor. In fact, there is also a tremendous potential for older spouses to pool their resources so that together they accomplish what would not be possible alone (anymore).

\section{Spousal Resources That Facilitate Healthy Lifestyles and the Mastery of Problems}

Another central tenet of this viewpoint is to address spousal resources that have the potential to boost physical activity and to buffer the negative impact of daily problems on stress in older spouses. We would like to specifically highlight the potential of collaborative problem solving and joint goals as two such spousal resources (fig. 1).

Collaborative problem solving can be defined by the extent to which two or more people appraise, represent, and solve a variety of tasks together (e.g., discussing problem solutions or pooling resources in joint action [29]). Experimental studies looking at collaborative problem solving in married couples have shown that older adults who collaborate with their spouse typically achieve better outcomes as compared to individual performance or when collaborating with a same-aged stranger [17]. Furthermore, collaborative problem solving also serves important compensatory functions, such as when one partner faces functional decline [30]. Field studies using repeated daily-life assessments in older couples have recently extended these findings by showing that collaborative problem solving, for example with regards to discussing problem solutions or acting jointly, is typically perceived as more effective across a variety of different daily problems than individual problem solving [31]. In other words, if an older adult can discuss his or her problem (e.g., regarding fear of falling) with their spouse, then he/she may be more likely to overcome the problem and leave the house. Older spouses who engage in collaborative problem solving may therefore be more successful in solving daily problems than older spouses who primarily use individual problem solving. Hence, collaborative problem solving may be a key spousal resource that buffers the negative association between daily problems, stress, and physical activity in older spouses.

Spousal goals may also help us better understand how spouses solve their problems. For example, previous research has shown that older adults who encountered problems regarding the socioemotional goals they share with their spouse were more likely to solve those problems using strategies that involve other people than older adults experiencing problems with individual goals [17, 32]. In other words, if spouses have joint goals (e.g., to attend their granddaughter's wedding), they may not only be more vulnerable to each other's daily problems, but they may also have a more vested interest in solving them collaboratively $[22,31]$. This reasoning is supported by recent couple research showing that older spouses with shared goals are particularly likely to engage each other in goal pursuit and also report frequent and enjoyable collaborations [22].

Future research will need to link this line of research with the earlier described biobehavioral pathways to ultimately answer the key question of what older couples might be able to do themselves and together as a team to 
embark on more favorable health trajectories for everyone involved. For example, it remains to be seen if collaborative problem solving is potent enough to buffer the well-reported negative association between the fear of falling and physical activity engagement [15]. Similarly, much more is known about the potential benefits of engaging in collaborative coping for mood and other selfreported outcomes [33] than for physiological outcomes such as salivary cortisol. Finally, it is an open question whether frequent spousal collaborations in daily life end up having downstream consequences because they ultimately impact long-term physical and mental health in old age. Answering such questions requires relatively complex designs that combine the strengths of daily-life assessments (e.g., using time-sampling methods) with long-term longitudinal follow-ups to track the proposed cumulative effects over time.

\section{Implications for Theory and Research}

The current viewpoint puts forward potential mechanisms that may explain previously documented spousal associations in long-term health trajectories. We provide a theoretical backdrop for the notion that it takes two to tango'. Specifically, we hope to inspire research on how older couples may engage in joint health behaviors (e.g., physical activity) and how such behaviors impact their own and joint health outcomes as well as instill a better understanding of what spouses may be able to do if one partner encounters difficulties (e.g., collaboratively solving the problem). As we look to the future, we see at least three promising areas of inquiry that would move the field forward.

\section{Social Models of Health Promotion}

More and more aging and health psychological scholars are recognizing the need to extend the well-established focus on individual health trajectories towards an inclusion of significant others such as spouses $[2,4,33]$. While there is a lot that remains to be learned about the psychological processes that may help set older spouses on more or less favorable health trajectories, marriage is but one interesting dyad to study. In fact, given different relationship patterns and changing family structures (particularly among future cohorts of older adults), more needs to be learned about the role relationship histories (e.g., relationship duration, previous divorce/widowhood) and partnership characteristics (heterosexual, gay; presence of children or not) have in shaping spousal health dynamics
[34]. Furthermore, other relationships beyond one's romantic partnership may also end up shaping health outcomes. For example, intergenerational relationships as well as friendship ties may provide complementary but different opportunities, as well as their own specific restrictions $[35,36]$.

\section{Incorporate Health Resources and Interpersonal Flourishing}

The social and medical sciences can look back on a long tradition of studying age-associated physical and mental health problems. In line with other lifespan scholars, we would like to propose that it may be time to move away from such deficit-oriented approaches and toward a much broader conceptualization of physical and mental health with aging [37]. For example, the WHO already defines health as 'a state of complete physical, mental and social well-being' [38]. According to this definition, it seems essential to look at mental health as also encompassing positive components such as personal growth, life satisfaction, positive emotional experiences, and interpersonal flourishing [37], and to address physical health by also identifying biological resources that undo the negative effects we know so much about (e.g., oxytocin buffering cortisol responses to stress) [28]. Ultimately, such an approach will help us to identify key resources and develop prevention and intervention programs that optimize aging outcomes and to tailor them to the respective social context.

\section{Moving Back and Forth between Lab and Life}

Technological and methodological innovations have played a major role in facilitating research that examines the daily-life processes that may accumulate over time and impact long-term health outcomes in individuals and couples [39]. Such approaches (e.g., time-sampling methods) provide a very detailed picture of the daily-life processes and the social dynamics that characterize married couples. One of the greatest strengths of time-sampling methods lies in the high ecological validity and immediate clinical relevance of findings [40]. However, timesampling research also has its limitations. For example, it is difficult to take into account, let alone control for the many factors that are involved in daily-life processes. Furthermore, time-sampling research provides a snapshot of interrelated daily processes, but it does not allow for causal inferences. It is thus important to go back and forth between lab and life to provide the best possible test of mechanisms assumed to underlie spousal interrelations in physical and mental health. Hence, in addition to choosing sophisticated ways of capturing daily-life pro- 
cesses that contribute to physical and mental health in couples, we need to go back to the laboratory and subject correlational findings to experimental testing under controlled laboratory conditions. Importantly, such a combination of methods may even be possible within the same study and involve the same spousal participants. For example, it is conceivable to combine a module of daily-life assessments of collaborative problem solving with a module that uses an experimental collaborative problem-solving paradigm $[30,31]$ within the same study.

\section{Conclusion}

This mini-review advances the notion that spousal health dynamics are much like a delicate dance that is shaped by a multitude of factors, some of which we are only beginning to understand. Such dynamics can neither be studied nor adequately understood without taking into account the perspectives of both partners. Moving forward, it will be important to study different combinations of individuals (e.g., friends vs. romantic dyads), to explore factors that make some dyads excel (e.g., specific resources and skills), thereby complementing a longstanding search for factors that cause problems, and to investigate the underlying dynamics as they unfold in real life and that end up impacting everyone involved.

\section{Acknowledgement}

Christiane Hoppmann gratefully acknowledges the support of the Michael Smith Foundation for Health Research and the Canada Research Chairs Program.

\section{References}

$>1$ Hoppmann C, Gerstorf D: Spousal interrelations in old age - a mini review. Gerontology 2009;55:459.

$\checkmark 2$ House JS, Landis KR, Umberson D: Social relationships and health. Science 1988;241:540545.

-3 Coyne JC, Rohrbaugh MJ, Shoham V, Sonnega JS, Nicklas JM, Cranford JA: Prognostic importance of marital quality for survival of congestive heart failure. Am J Cardiol 2001; 88:526-529.

4 Berg CA, Upchurch R: A developmental-contextual model of couples coping with chronic illness across the adult life span. Psychol Bull 2007;133:920-954.

$\checkmark 5$ Hoppmann C, Gerstorf D, Hibbert A: Spousal associations between functional limitations and depressive symptom trajectories: longitudinal findings from the study of Asset and Health Dynamics among the Oldest Old (AHEAD). Health Psychol 2011;30:153-162.

6 Strawbridge WJ, Wallhagen MI, Shema SJ: Impact of spouse vision impairment on partner health and well-being: a longitudinal analysis of couples. J Gerontol B Psychol Sci Soc Sci 2007;62B:S315-S322.

$\checkmark 7$ Schwarzer R: Modeling health behavior change: how to predict and modify the adoption and maintenance of health behaviors. Appl Psychol Int Rev 2008;57:1-29.

8 Brassington GS, Hekler EB, Cohen Z, King AC: Health-enhancing physical activity; in Baum A, Revenson TA (eds): Handbook of Health Psychology. Hoboken, Psychology Press, 2012, pp 353-374

$\checkmark$ Blair SN: Physical inactivity: the biggest public health problem of the 21 st century. $\mathrm{Br} J$ Sports Med 2009;43:1-2.
10 US Department of Health and Human Services: Physical Activity Guidelines for Americans. Atlanta, Center for Disease Control, 2008.

$>11$ Hoppmann CA, Gerstorf D, Luszcz M: Spousal social activity trajectories in the Australian Longitudinal Study of Ageing in the context of cognitive, physical, and affective resources. J Gerontol B Psychol Sci Soc Sci 2008;63:P41P50.

12 Burkert S, Scholz U, Gralla O, Roigas J, Knoll $\mathrm{N}$ : Dyadic planning of health-behavior change after prostectomy: a randomized-controlled planning intervention. Soc Sci Med 2011;73:783-792.

13 Lent RW, Lopez FG: Cognitive ties that bind: a tripartite view of efficacy beliefs in growthpromoting relationships. J Soc Clin Psychol 2002;21:256-286.

14 Bussmann JBJ, Ebner-Priemer UW, Fahrenberg J: Ambulatory activity monitoring progress in measurement of activity, posture, and specific motion patterns in daily life. Eur Psychol 2009;14:142-152.

15 Yardley L, Smith H: A prospective study of the relationship between feared consequences of falling and avoidance of activity in community-living older people. Gerontologist 2002;42: 17-23.

16 Kliegel M, McDaniel MA, Einstein GO: Plan formation, retention, and execution in prospective memory: A new approach and agerelated effects. Mem Cognit 2000;28:10411049.

17 Berg CA, Strough J: Problem solving across the life span; in Fingerman K, Berg CA, Antonnuci T, Smith J (eds): Handbook of LifeSpan Psychology. New York, Springer, 2011, pp 239-267.
18 Piazza JR, Almeida DM, Dmitrieva NO, Klein LC: Frontiers in the use of biomarkers of health in research on stress and aging. J Gerontol Psychol Sci Soc Sci 2010;65:513525.

19 Seeman TE, Gruenewald TL: Allostasis and allostatic load over the life course; in van Lange PAM (ed): Medical and Psychiatric Comorbidity over the Course of Life. Washington, American Psychiatric Publishers, 2006, pp 179-196.

20 Miller GE, Chen E, Cole SW: Health psychology: developing biologically plausible models linking the social world and physical health. Ann Rev Psychol 2009;60:1-24.

21 Carstensen LL, Fung HH, Charles ST: Socioemotional selectivity theory and the regulation of emotion in the second half of life. Motiv Emot 2003;27:103-123.

22 Schindler I, Berg CA, Butller JM, Fortenberry KT, Wiebe DJ: Late-midlife and older couples' shared possible selves and psychological well-being during times of illness: the role of collaborative problem solving. J Gerontol B Psychol Sci Soc Sci 2010;65:416-424.

$\checkmark 23$ Larson RW, Almeida DM: Emotional transmission in the daily lives of families: a new paradigm for studying family process. J Marriage Fam 1999;61:5-20.

24 Robles TF, Slatcher RB, Trombello JM, McGinn MM: Marital quality and health: a metaanalytic review. Psychol Bull 2014;140:140187.

25 Kumsta R, Heinrichs M: Oxytocin, stress and social behavior: neurogenetics of the human oxytocin system. Curr Opin Neurobiol 2013; 23:11-16 
26 Ditzen B, Schaer M, Gabriel B, Bodenmann G, Ehlert U, Heinrichs M: Intranasal oxytocin increases positive communication and reduces cortisol levels during couple conflict. Biol Psychiatry 2009;65:728-731.

27 Huffmeijer R, Ijzendoorn MH, BakermansKranenburg MJ: Ageing and oxytocin: a call for extending human oxytocin research to ageing populations - a mini-review. Gerontology 2013;59:32-39.

28 Klaperski S, Dawans B, Heinrichs M, Fuchs R: Does the level of physical exercise affect physiological and psychological responses to psychosocial stress in women? Psychol Sport Exerc 2013;14:266-274.

-29 Dixon RA, Fox DP, Trevithick L, Brundin R: Exploring collaborative problem solving in adulthood. J Adult Dev 1997;4:195-208.

30 Rauers A, Riediger M, Schmiedek F, Lindenberger U: With a little help from my spouse: does spousal collaboration compensate for the effects of cognitive aging? Gerontology 2011;57:161-166.

31 Hoppmann C, Gerstorf D: Spousal goals, affect quality, and collaborative problem solving: evidence from a time-sampling study with older couples. Res Hum Dev 2013;10:70-87.

32 Hoppmann C, Heckman-Coats A, BlanchardFields F: Goals and everyday problem solving: examining the link between age-related goals and problem-solving strategy use. Neuropsychol Dev Cogn B Aging Neuropsychol Cogn 2008; 15:401-423.

33 DeLongis A, Holtzman S: Coping in context: the role of stress, social support, and personality in coping. J Pers 2005;73:1633-1656.

34 Balsam KF, A'Augelli AR: The victimization of older lesbian, gay, bisexual, and transgender adults: patterns, impact, and implications for intervention; in Kimmel D, Rose T, David $S$ (eds): Lesbian, Gay, Bisexual, and Transgender Aging: Research and Clinical Perspec- tives. New York, Columbia University Press, 2006, pp 110-130.

35 Fingerman KL, Sechrist J, Birditt K: Changing views on intergenerational ties. Gerontology 2013;59:64-70.

36 Fuller-Iglesias HR, Webster NJ, Antonucci TC: Adult family relationships in the context of friendship. Res Hum Dev 2013;10:184203.

37 Ryff CD, Singer B: Interpersonal flourishing: a positive health agenda for the new millennium. Pers Soc Psychol Rev 2000;4:30-44.

38 World Health Organization: Official Records No. 2. New York, WHO, 1948.

39 Hoppmann CA, Riediger M: Ambulatory assessment in lifespan psychology: an overview of current status and new trends. Eur Psychol 2009;14:98-108.

40 Bolger N, Davis A, Rafaeli E: Diary methods: capturing life as it is lived. Ann Rev Psychol 2003;54:579-616. 